Article

\title{
All Sizes Fit the Red Queen
}

\author{
Indré Žliobaité (D) and Mikael Fortelius
}

\begin{abstract}
The Red Queen's hypothesis portrays evolution as a never-ending competition for expansive energy, where one species' gain is another species' loss. The Red Queen is neutral with respect to body size, implying that neither small nor large species have a universal competitive advantage. Here we ask whether, and if so how, the Red Queen's hypothesis really can accommodate differences in body size. The maximum population growth in ecology clearly depends on body size-the smaller the species, the shorter the generation length, and the faster it can expand given sufficient opportunity. On the other hand, large species are more efficient in energy use due to metabolic scaling and can maintain more biomass with the same energy. The advantage of shorter generation makes a wide range of body sizes competitive, yet large species do not take over. We analytically show that individuals consume energy and reproduce in physiological time, but need to compete for energy in real time. The Red Queen, through adaptive evolution of populations, balances the pressures of real and physiological time. Modeling competition for energy as a proportional prize contest from economics, we further show that Red Queen's zero-sum game can generate unimodal hat-like patterns of species rise and decline that can be neutral in relation to body size.
\end{abstract}

Indre Žliobaite. Department of Computer Science, University of Helsinki, Helsinki 00013, Finland; Finnish Museum of Natural History, Helsinki 00100, Finland. E-mail: indre.zliobaite@helsinki.fi

Mikael Fortelius. Department of Geosciences and Geology, University of Helsinki, Helsinki 00014, Finland; Finnish Museum of Natural History, Helsinki 00100,Finland. E-mail: mikael.fortelius@helsinki.fi

Accepted: 31 July 2020

\section{Introduction}

One of the most influential evolutionary theories-the Red Queen's hypothesis (Van Valen 1973, 1980)—portrays species evolution as a never-ending competition for expansive energy,* where one species' gain inevitably results in a corresponding loss for other species. Energy production and consumption in organisms is governed by metabolism, and metabolic scaling characterizes relationships between energy and body size (Schmidt-Nielsen 1997). Despite recent progress (Damuth 2007), how macroevolutionary and metabolic theories fit together remains an open question.

\footnotetext{
*Expansive energy is energy used for growth and reproduction (Van Valen 1976). It can be used as common currency to quantify fitness across various organisms and different body sizes. Maximizing expansive energy rather than minimizing total energy consumption matters. Trophic energy by individual = productive energy + waste energy + structural energy (earlier production) + reserve energy. Productive energy = maintenance energy + expansive energy.
}

Body size is perhaps the most widely researched functional trait in paleobiology (Damuth and MacFadden 1990). Many empirical patterns of spatial and temporal distributions of body mass have been identified and debated in various ecological circumstances (e.g., Bergman's rule, Cope's rule). Not surprisingly, body size has been portrayed as one of the most direct links between microevolution and macroevolution (Maurer et al. 1992; Jablonski 1996). Indeed, almost inevitably, different body sizes will carry competitive advantages in different ecological circumstances (Brown and Maurer 1986; Woodward et al. 2005; White et al. 2007; Bribiesca et al. 2019). A widely debated but still open question is whether any particular body sizes carry universal competitive advantages across all possible ecologies.

Metabolic theory implies that large animals are more efficient in energy consumption, because they use less energy per unit of body mass (Kleiber 1932). Many life-history parameters, such as life span, generation length, number of offspring, and even durability of 
teeth are tightly linked to metabolic scaling (Schmidt-Nielsen 1984; Fortelius 1985). Yet the Red Queen's hypothesis implies* that spectra of body sizes can be competitive within adaptive zones, ${ }^{\dagger}$ that is, no particular body size carries an overarching evolutionary advantage. Are there evolutionary mechanisms that allow large and small species to compete as equals within the Red Queen's realm?

Large species can support more biomass than small species within a constant amount of energy (Kleiber 1932; Schmidt-Nielsen 1984). If large and small species are indeed equally competitive, but large species are more efficient in energy use, why do large species not take over? Or is their tendency to do so in fact what we see in Cope's rule?

\section{The Red Queen Meets Cope's Rule}

Cope's rule is a widely recognized yet extensively debated empirical generalization of macroevolutionary trends, also known as Alroy's axiom (Polly 1998), or even Marsh's maxim (Raia and Fortelius 2013). The pattern refers to the tendency for species within a clade (Cope would have said "lineage") to evolve toward a larger body size (Alroy 1998; Clauset and Erwin 2008; Huang et al. 2017). While the mechanisms behind apparent body-size trends are still actively debated (Jablonski 1997; Hone and Benton 2005; Raia et al. 2012; Pineda-Munoz et al. 2016), it is clear that specialization alone does not provide a sufficient explanation for patterns attributed to Cope's rule. While the debate of ecological circumstances continues, an overarching question remains open: To what extent does body size itself, disregarding dietary or climatic specializations, carries any universal selective advantage?

*The Red Queen's hypothesis does not postulate that the amount of energy is invariant to body mass, but it must imply it. Van Valen (1971, 1973) did not explicitly discuss body mass in the Red Queen's hypothesis or the notion of an adaptive zone; the implication clearly follows from the lack of explicit constraints and conditions, for instance, that the hypothesis would only hold for taxa of the same size.

${ }^{\dagger}$ An adaptive zone (Simpson 1944, 1953; Van Valen 1971) here roughly means that species are competing for the same resources. A more detailed account on the history and interpretation of the term is given in Appendix 1.
The theoretical implications of fast versus slow life histories that underlie the concept of $\mathrm{r} / \mathrm{K}$ selection (MacArthur and Wilson 1967) and related constructs might offer a partial explanation. $\mathrm{r} / \mathrm{K}$ selection postulates a gradient between two extreme types of species: r-selected species are small and have high growth rates. They live in less crowded but unpredictable environments, where the ability to reproduce rapidly is important. K-selected species, in contrast, are larger, occupy more stable environments, and live at densities close to the carrying capacity of their environments (Pianka 1970; Cunningham et al. 2001; Reznik et al. 2002; Brown et al. 2004). r/K selection posits that when resource exploitation approaches the limit of the carrying capacity, the prevailing adaptive strategies change from $r$ toward K (Southwood et al. 1974; Hallam 1978), pushing large, slow-reproducing species to become dominant in that environment (Dobson and Oli 2008; Salguero-Gómez et al. 2016). Plausible as it is, this mechanism does not explain why large K-selected species do not completely replace smaller r-selected species as unsaturated, low-competition habitats become saturated.

A common empirical pattern of body-size evolution within clades is an expansion in the size range rather than in average size. Several decades ago Stanley (1973) had already pointed out, in the context of Cope's rule, citing a 1968 paper by Bonner, that small species do not vanish; instead, large species become more common over the duration of a clade. The theoretical nicety of whether new species within a clade arise via anagenesis or cladogenesis is largely unknowable in practice but fortunately does not matter for this argument, which only depends on the relative frequencies observed. An asymmetric expansion in body-size range will inevitably show an increase in average body mass within the clade, as Jablonski (1997) empirically demonstrated for some Cretaceous mollusks. Gillman (2007) generalized this to vertebrate body mass ranges and showed that body mass ranges in morphologically disparate clades expand in highly predictable ways over time. We ask what evolutionary mechanisms could explain this.

One possible explanation is that the Red Queen's pressure is not equally prevalent 
throughout the domain of Cope's rule, but her jurisdiction is stronger toward the end of the pattern. As higher taxonomic clades usually start with some major phenotypic innovation, this temporarily makes resources less limited.* With less-limited resources, small species can expand faster than large species (due to shorter generation length; Brown 1995). Transitioning from relatively unlimited to fully exploited resources (the Red Queen's zero-sum game) dilutes the advantage of shorter generation length and makes a wide range of body masses equally competitive, as we will demonstrate numerically with a coarse algebraic model. Yet, while individuals consume energy and reproduce in physiological time, competition for energy across species must happen in real time. Operating in both physiological and real time is the Red Queen's challenge. She balances the two times, operating via species (or metapopulations) as genetically cohesive units of adaptive evolution.

We propose that body size has no universal evolutionary advantage in the Red Queen's domain, because expansive energy is acquired so slowly under zero-sum game conditions that it makes generation length irrelevant. Imagine that the government gives unlimited money for building houses, but the next house can be started only when the previous one is finished. Under such circumstances, those who build faster will benefit more. But if the wait until the government disburses the money is very long, say 10 years, everybody will have finished their first house by then, and it does not matter who was faster. That is why, in the Red Queen's domain, small species do not take over.

Maintaining a gram of body tissue in real time is cheaper for large animals (metabolic scaling; Kleiber 1932), but the cost of maintaining $1 \mathrm{~g}$ of body tissue over an individual organism's lifetime is the same for all body masses (scaling of life expectancy; Lindstedt and Calder 1981). Large animals, therefore, can maintain more biomass than small animals

*Prevalence of empty niches and consequences thereof to species diversity have been extensively considered in the equilibrium models of species diversity (Walker and Valentine 1984). An adaptive zone is conceptually close, but not the same as a niche. An adaptive zone only exists when it is occupied. with a constant amount of energy, but this does not help to accelerate expansion of population biomass. That is why large species do not take over in the Red Queen's domain.

To analyze the mechanism from the ecological perspective, we model the Red Queen's competition for energy as a proportional prize contest from economics (Cason et al. 2020), in which rewards are shared in proportion to performance. We show that under the assumption that acquisition of an evolutionary advantage (e.g., extra cutting crest on a tooth) does not depend on body mass, the expected effect of such an evolutionary step on population growth rates does not depend on body mass. The question is not trivial, because individuals' food intake depends on body size and scales allometrically with it (Shipley et al. 1994).

Modeling the Red Queen's competition as a proportional prize contest can generate macroevolutionary hat-like patterns of unimodal rise and decline (Jernvall and Fortelius 2004; Foote et al. 2007; Liow and Stenseth 2007; Zliobaite et al. 2017). Our computational experiments demonstrate that in the absence of environmental change, unimodal hat-like patterns can be expected due to random origination of new species. While Cope's rule arises from acrossspecies competition, the hat-like patterns can arise from within-species competition when transitioning from a relatively unlimited resource state to a zero-sum game. The remainder of the paper analyzes the evolutionary mechanics and implications of this process.

\section{Metabolic Rate and Expansive Energy}

Body mass effectively determines the pace of life (Schmidt-Nielsen 1984), and vice versa. Metabolic scaling establishes a fundamental difference in energy allocation: large organisms are relatively more efficient in maintenance, but slower in reproduction (Brown 1995). Terrestrial mammals span 24 orders of magnitude in body mass from shrews weighing $2 \mathrm{~g}$ to elephants weighing 5 metric tons today, or even 15 metric tons or more in the past (Smith et al. 2010), showing that various body sizes can be viable. Macroevolutionary theory, particularly the Red Queen's hypothesis, postulates a zerosum game for energy across species, implicitly, 
species of any size. Control of energy is not simply about acquiring it once; survival requires a process of repetitive acquisition. Thus, competition as such is not for energy, but for access to energy. And if energy is acquired by outcompeting other species, the question arises how a larger, more energy-efficient species does not gain relatively more control of resources from a smaller species' loss.

Metabolic Scaling.-The dominant metabolic scaling relation, established by Kleiber (1932), is roughly $W \propto M^{0.75}$, where $W$ is energy consumption rate and $M$ is body mass. ${ }^{*}$ Different technical explanations for the scaling exponent have been evoked (Glazier 2014), ranging from supply networks (West et al. 1997; Brown et al. 2004; Savage et al. 2008) to thermodynamics (Ballesteros et al. 2018). Some authors have argued that there is no universal metabolic scaling exponent, only ranges that give the upper and lower bounds (Banavar et al. 2010; Glazier 2014; White and Kearney 2014; Virot et al. 2017).

Even though measured empirical exponents vary considerably within and among species, depending on ecological factors as well as developmental and physiological status (White and Kearny 2014; Harrison 2017; Glazier 2018), it is clear that the metabolic rate of animals cannot scale isometrically (DeLong et al. 2010). ${ }^{\dagger}$ Organisms fuel a threedimensional body while their interactions with the environment are predominantly twodimensional. Feeding is on three-dimensional objects that are typically collected over a twodimensional area, using locomotion, which is effectively one-dimensional. By and large, all mammalian organs, except for the heart, operate via surfaces (Schmidt-Nielsen 1997). Transportation within an organism's vascular network is effectively one-dimensional. Thus, organisms often operate at a combination of three-, two-, and one-dimensional spaces. The

\footnotetext{
*The metabolic scaling exponent is still debated. In Appendix 2, we generalize the scaling argument using metabolic rate $M^{x}$, where one can plug in one's preferred exponent $x$. For clarity of exposition in the main text, we use $M^{0.75}$.

Unless they are colonial or change shape with size. These are special cases, which for clarity we leave out of this analysis.
}

dimensionality mismatch between interfaces for energy acquisition, energy transportation within the body, and energy consumption is the fundamental reason why metabolic rate has to slow down with body-size increase.

Yet the Red Queen implies that the amount of energy controlled by each species is invariant with body mass. We take this as a working assumption and search for the simplest ecological and evolutionary mechanisms that could potentially permit such a balance.

With nonisometric metabolic scaling, the number of individuals within a species that can be supported by a fixed amount of energy is also nonisometric in relation to body size. If one individual consumes energy at rate $W \propto$ $M^{0.75}$ (e.g., joules per day), then the number of individuals that can be supported with constant amount of energy $\left(E \propto M^{0}\right)$ scales as $N=E / W \propto M^{0} / M^{0.75}=M^{-0.75}$.

Ample empirical evidence suggests that the population density of primary consumers globally scales close to $\mathrm{M}^{-0.75}$ (Damuth 1981; Marquet 2002; Jetz et al. 2004). This empirical pattern, often referred to as the energy equivalence rule (Nee et al. 1991; Marquet et al. 1995), has been shown to still hold for mammalian primary consumers when the data are broken down by geographic area, by broad habitat type, and by individual community (Damuth 1987). This pattern, consistent with Van Valen's Red Queen, suggests that "species in general should not differ in their potential for extracting energy from the environment solely as a function of their size" (Ginzburg and Colyvan 2004: loc. 1281). Using simulation models, Damuth (2007) demonstrated that when randomly chosen species evolve to take energy from other species, population densities settle at an inverse of the metabolic rate $\left(n \propto M^{-0.75}\right)$. Yeakel et al. (2018) demonstrated a mechanism for this scaling of population densities (as well as implications for Cope's rule) by modeling population dynamics from the starvation perspective. Nonetheless, a synthesis of micro- and macroevolutionary mechanisms in the context of the Red Queen is still lacking.

Some empirical evidence suggests more shallow scaling of population densities (May 1988; Pedersen et al. 2017; Stephens et al. 2019), which would challenge the energetic 
equivalence. ${ }^{*}$ Researchers have argued that the energetic equivalence rule is more an upper limit (Marquet et al. 1995) than an average expected relationship. Because there are generally more small species than large species (Polishchuk and Blanchard 2019), mismatch in species history stages or saturation of an ecosystem with respect to the carrying capacity may lead to larger deficits at smaller body sizes and thus drive the empirical population density exponent closer toward zero. In our analysis we consider the upper-limit exponent and, assuming maximally intense competition, we analyze how the expansion of populations scales.

Energy for Population Growth.-Population growth needs two main ingredients - time and energy. If unlimited energy for expansion is available, generation length (time) is the limiting factor for population growth. This is well known from ecology, where theory and empirical observations suggest that the maximum population growth rate scales as $r_{\text {growth }}^{\max } \propto$ $M^{-0.25}$ (Fenchel 1974; Brown 1995), where $M$ is body mass. The negative exponent implies that, given enough resources, small species can expand their populations faster.

In the Red Queen's domain, expansive energy becomes available for species in chunks that are independent of body mass, $E_{\text {expansive }}^{\text {RedQ }} \propto M^{0}$. This argument directly builds on Van Valen's reasoning from a single-species perspective. "To a good approximation, each species is part of a zerosum game against other species. Which adversary is most important for a species may vary from time to time, and for some or even most species no one adversary may ever be paramount. Furthermore, no species can ever win, and new adversaries grinningly replace the losers." (Van Valen 1980: p. 294). Damuth (2007) modeled this computationally, arguing that, in the Red Queen's domain, the potential for an "evolutionary advance" of a species does not depend on its body mass.

*Polishchuk and Blanchard (2019) provided evidence for a biomass equivalence rule; they did not deal with an energy equivalence rule. The biomass equivalence rule is for all organisms of given size classes; the energy equivalence rule is for species. Therefore, biomass equivalence translates to the energy equivalence rule as long as the number of species scales as $M^{-0.25}$.
Population growth rate over a time period is defined as $r_{\text {growth }}=N^{*} / N-1$, where $\mathrm{N}$ is the number of individuals in the previous time step, and $N^{*}$ is the number of individuals now. The expansive energy required to support population growth is $E_{\text {expansive }}=\left(N^{*}-N\right) W$, where $W$ is the individual metabolic rate. Using the theoretical and empirical observation that population size (density) scales as the inverse of metabolic rate $\left(N \propto W^{-1}\right)$, we expansive energy proportional to the population growth rate, $E_{\text {expansive }} \propto r_{\text {growth }}$.

From here, to sustain the maximum physiological growth rate, expansive energy would need to scale as $E_{\text {expansive }}^{\max } \propto r_{\text {growth }}^{\max } \propto M^{-0.25}$. This means that in order to sustain maximum population growth, populations of small species would need to be able to acquire consistently more expansive energy per unit time than large species. However, if expansive energy becomes available in small chunks independently of body mass, smaller species cannot acquire consistently more energy, and the population growth rate is expected to be independent of body mass, $r_{\text {growth }}^{\text {RedQ }} \propto E_{\text {expansive }}^{\max } \propto M^{0}$.

Demographic Paths to Evolutionary Expansion.For completeness of the scaling argument, we need to consider in what ways expansive energy can translate into a larger population, that is, how evolutionary and ecological perspectives connect. Expansive energy does not generate individuals directly, but recurring access to that energy can support a larger population of individuals. For a population to grow, more individuals need to be born than die. ${ }^{\dagger}$ Thus, expansive energy would need to increase population birth rates, reduce mortality rates, or both. Let us consider possible mechanisms for this to happen.

The scaling of birth and mortality rates, as for most life-history descriptors, closely relates to metabolic scaling (Lindstedt and Calder 1981; Brown et al. 2004; Hulbert et al. 2007). This is

\footnotetext{
${ }^{\dagger}$ If the birth rate across a population is $25 \%$ and the death rate is $20 \%$, the population will grow $5 \%$ per period. This follows from the definition of growth rate $r_{\text {growth }}=N^{*} / N-1=(N+$ $\left.N_{\text {born }}-N_{\text {died }}\right) / N-1=N_{\text {born }} / N-N_{\text {died }} / N=r_{\text {birth }}-r_{\text {death }}$ where $N$ is the number of individuals at the previous time step, $N_{\text {born }}$ is the number of individuals born during this period, $N_{\text {died }}$ is the number of individuals that died during this period, and $r_{\text {birth }}$ and $r_{\text {death }}$ are the population birth and mortality rates, respectively.
} 
generally known as the rate of living theory, which postulates that life span is inversely related to metabolic rate (Pearl 1928). As for many other scaling relationships, the theory for rate of living is still incomplete, and the exponents are debated (Speakman 2005; de Megalhaes et al. 2007; Glazier 2015). Despite debates about the details, the general relationships are clear-smaller animals mature and reproduce faster and die sooner. At the same time, allowing for individual nuances, animals experience roughly the same number of heartbeats, breaths (Peters 1983), or chews (Fortelius 1985) per lifetime, independent of their body mass. Thus, the durability of an organism (how many heartbeats, breaths, chews) scales as $M^{0}$.

During an animal's lifetime, a gram of body tissue expends about the same amount of energy whether the tissue is in a guinea pig, cat, dog, cow, or horse (Rubner 1908). Thus, energy consumption per day per gram of body tissue scales as the metabolic rate of the whole organism divided by its mass $\left(M^{0.75} / M^{1}=\right.$ $\left.M^{-0.25}\right)$. The maximum life span of an organism then must scale as its durability divided by the speed of use $\left(M^{0} / M^{-0.25}=M^{0.25}\right)$.

Maximum life span defines physiological lifetime if no misfortunes happen. Life span that includes ecological risks is called "ecological longevity" and is described statistically as life expectancy at birth. Scaling literature for animals often reports either one or the other, but both are expected and reported to scale with body mass with the same theoretical exponent of 0.25 (Polishchuk and Tseitlin 1999).

Under a coarse assumption of uniform mortality rate across the age groups, ${ }^{*}$ mortality rate is equal to the inverse of ecological life expectancy (Keyfitz and Caswell 2005). In such a case, considering that life expectancy scales as $M^{0.25}$, mortality rates should correspondingly scale as $r_{\text {death }} \propto M^{-0.25}$. The empirical mortality rates of mammals indeed have been reported to scale very closely to this,

\footnotetext{
*This assumes that the mortality rate is constant across the age groups. In reality, young and old individuals of a natural population are generally more likely to die than middle-aged individuals (Tidiere et al. 2016), but it is a useful approximation commonly taken to allow generic demographic analysis (Keyfitz and Caswell 2005).
}

with the exponent of -0.24 (McCoy and Gillooly 2008).

When a population does not grow, birth rates must scale with the same exponent as mortality rates $r_{\text {birth }}=r_{\text {death }} \propto M^{-0.25}$. Indeed, annual fecundity ${ }^{\dagger}$ of mammals has been observed to scale with the exponent of -0.26 (Hamilton et al. 2011).

When a population grows, birth rates exceed death rates. An increase in birth rates would require either more frequent births or a greater number of offspring per birth per reproductive female. While specialized life-history strategies are widely recognized (Sibly and Brown 2009), birth rates are primarily governed by metabolic scaling (Lindstedt and Calder 1981) and are not easy to change permanently without physiological trade-offs. Elevated reproductive rates lead to shortened life span, as limited resources enforce a compromise between investment in reproduction and somatic maintenance (Kirkwood 1977). All else being equal, permanent and continuous increase in birth rate thus seems unlikely to be the main path of evolutionary expansion.

A more straightforward way for populations to grow is to increase average life expectancy. Increased life expectancy does not imply extending the life span of the oldest individuals, but rather adding more time throughout all age classes. Such effects have been observed by Tidiere et al. (2016), who compared mortality rates of zoo and wild animals and showed that being at a zoo reduces the mortality rates of all age groups by a roughly constant factor. An evolutionary advance ${ }^{\ddagger}$ could reduce mortality rates by reducing health risks, exposure to predators or risks of accident by walking faster or shorter distance to the next food item; higher probability of finding food; or higher chewing or digestion efficiency. For example,

\footnotetext{
${ }^{\dagger}$ Annual fecundity is reported per female, while the birth rate is defined per population, but because the number of reproducing females is proportional to the total population size (Polishchuk and Tseitlin 1999), these two rates are bound to have the same scaling exponent.

*An advance here has a broader meaning than an innovation would. An innovation would imply something globally new, an advance may be new for that particular species only, it may, in boundary cases, be even something that a clade lost earlier and then reacquired.
} 
Van Valkenburgh (2009) demonstrated that the risk of tooth fracture for predators increases when competition is tighter. If an evolutionary advance can temporarily (say, until competitors catch up) work in a way conceptually analogous to supplementary feeding of a favored wild species or protection of a domestic one from predators, it would support population growth and, in turn, evolutionary expansion.

Our next step is to model mechanisms of how an evolutionary advance translates to expansive energy and analyze conditions under which the acquired expansive energy at the population level may or may not depend on body size. This question is important for understanding conditions under which large and small species can compete as equals, despite individuals having different daily energy efficiencies (metabolism).

\section{Connecting Micro and Macro: Evolution under the Red Queen as a Proportional Prize Contest}

We model the relationships between individual evolutionary advantage and gain in expansive energy by a population of species as a proportional prize contest known from economics (Cason et al. 2020), in which rewards are shared in proportion to performance. The winning species do not gain all the available resources instantaneously; the gain is only proportional to the competitive advantage(s) that they have acquired.

An evolutionary advantage (the outcome of an advance in a sense of Damuth [2007]) is a newly acquired physical, physiological, or behavioral trait of a species that increases its advantage, measured as the probability of survival of individuals in a given adaptive zone at a given time. Such a trait can be, for example, an extra cutting crest on a molar tooth. The effectiveness of a particular trait depends on the environmental context; for example, in some circumstances, an extra crest may be beneficial, in others neutral, while having an extra crest may even be disadvantageous under certain circumstances.

Modeling Assumptions.-As in most computational modeling, our model builds on a set of assumptions about evolutionary processes, which often are simplifications, but necessary to help clarity of exposition. While the real world accommodates many more nuances and circumstances, our purpose here is to demonstrate that such a basic ecological process can, at least in principle, by itself generate macroevolutionary patterns (such as hat patterns), without separate, macrolevel explanations.

We assume that an evolutionary advantage at an individual level can arise with a probability that is independent of body mass, metaphorically, a mouse and an elephant are equally likely to acquire an advantage. We also assume that large and small species are likely to experience mutations leading to a particular trait with the same probability, although we are aware that in reality, the probability of acquiring a new trait has many factors, and various interdependencies are likely to occur at the genomic level, including different genome sizes and the fact that the same traits may be encoded differently in different organisms (Loewe and Hill 2010).

We further assume that species exist as evolutionary units (Simpson 1961), that is, that every individual within the species shares the same trait. More precisely, we assume that within-species variation is less than betweenspecies variation. This is typically the case for traits that identify fossil species, even though the maximum range of between-species differences may overlap (Polly et al. 2017). We then assume that the new trait provides a competitive advantage in energy acquisition. This can manifest itself as walking faster or requiring shorter distances to reach the next food item (due to access to different dietary items); higher probability of finding food; higher chewing or digestion efficiency; or more effective escape from predators, allowing longer or more concentrated periods of feeding. Not all morphological traits would fall under this assumption. For example, an increase in dental crown height would extend the durability of teeth but would not directly increase effectiveness of day-to-day resource acquisition. Finally, we assume that the evolutionary advantage is such that it does not change the body mass, does not extend life span, and does not directly give more offspring and that the individual metabolic rate stays the same. Within the scope of this study, we assume only one trophic 
level. We also assume that no external environmental changes take place.

We conceptualize the proportional prize competition with the following model: our analysis assumes a closed environment of a finite carrying capacity, which remains fixed during the period of analysis. The model assumes that within every time period (e.g., a day), a constant amount of energy is generated in the environment. Individuals start eating according to their metabolic speeds and eat until food available on that day runs out. The model assumes that energy comes in a standardized form, that all individuals can extract the same amount of energy from a unit of food, and that all individuals have the same amount of time available for eating. We model the efficiency of resource acquisition as the average speed of food acquisition throughout the period. The increase in efficacy of resource acquisition does not necessarily mean instantaneous acceleration of intake (eating faster), but rather that less time per day must be spent on foraging (finding food faster).

Given the model, we ask how the species' gain in expansive energy over time will depend on body mass.

Scaling of Expansive Energy for Evolving Species. - One might think that the question is trivial, because biomechanical effectiveness of acquired traits does not depend on body mass, so the gain should not depend on it either. Yet food intake of individuals depends on body size and scales allometrically with it (Shipley et al. 1994). Analytically, this imbalance resolves as noted in the following paragraphs.

Consider two species, which at the initial time step are at equilibrium-neither species is expanding or declining. Let $W_{\mathrm{A}}$ be the metabolic rate of species $\mathrm{A}$, and $W_{\mathrm{B}}$ be the metabolic rate of species $B$. Let us define the initial speed of food acquisition to be proportional to the metabolic rate $S_{\mathrm{A}} \propto W_{\mathrm{A}}$ and $S_{\mathrm{B}} \propto W_{\mathrm{B}}$. Let the number of individuals of the two species be $N_{1}$ and $N_{2}$. Let $T$ denote the fraction of day that both species spend on foraging. Then the trophic energy controlled by each population is $E_{\mathrm{A}}=N_{\mathrm{A}} S_{\mathrm{A}} T$ and $E_{\mathrm{B}}=N_{\mathrm{B}} S_{\mathrm{B}} T$. The carrying capacity of the environment is then the sum of energies controlled by the populations of the two species $K=E_{\mathrm{A}}+E_{\mathrm{B}}$.
Suppose species A acquires an evolutionary advantage as $\alpha>1$, such that the speed of food acquisition for this species becomes $S_{\mathrm{A}}^{*}=\alpha S_{\mathrm{A}}$. Assume that the second species does not evolve any new traits, and thus its speed of eating remains the same, that is, $S_{\mathrm{B}}^{*}=S_{\mathrm{B}}$.

Because species $A$ has evolved an advantage and can now acquire energy faster, the total amount of energy that can be acquired per individual during the next period changes. The equilibrium state provides just enough food for all individuals, but when species A speeds up food acquisition, the daily carrying capacity of the environment is exhausted faster. Individuals of species A become slightly better nourished, while individuals of species B become slightly undernourished.

The foraging time for both species thus becomes $T^{*}=\beta T$, where $\beta<1$ is a coefficient that depends on the initial population sizes of both species. From the assumption that the carrying capacity of the environment stays constant, we can find the expression for $\beta$.

Assuming that the number of individuals stays the same throughout the period, the amount of energy obtained by species A during the period is:

$$
E_{\mathrm{A}}^{*}=N_{\mathrm{A}} S_{\mathrm{A}}^{*} T^{*}=N_{\mathrm{A}} \alpha S_{\mathrm{A}} \beta T=\alpha \beta E_{\mathrm{A}} .
$$

The amount of energy obtained by species B is

$$
E_{\mathrm{B}}^{*}=N_{\mathrm{B}} S_{\mathrm{B}}^{*} T^{*}=N_{\mathrm{B}} S_{\mathrm{B}} \beta T=\beta E_{\mathrm{B}} .
$$

Consider that the carrying capacity stays the same, that is, $K=E_{\mathrm{A}}+E_{\mathrm{B}}=E_{\mathrm{A}}^{*}+E_{\mathrm{B}}^{*}$. From these equations we can express the multiplier for the new foraging time as

$$
\beta=\left(E_{\mathrm{A}}+E_{\mathrm{B}}\right) /\left(\alpha E_{\mathrm{A}}+E_{\mathrm{B}}\right) .
$$

We denote the proportion of energy controlled by species A initially as $\pi=E_{\mathrm{A}} / K$. Then, we get

$$
\beta=1 /(\alpha \pi+1-\pi) .
$$

Species A population will now control energy in excess of its equilibrium metabolic rate. This can be thought as the extra energy that improves the probability of survival and allows 
the population to grow, as analyzed in the previous section. It remains to analyze how the population growth rate depends on the evolutionary advantage $\alpha$.

The amount of energy obtained by species A with the new trait can support $N_{\mathrm{A}}$ * individuals, where

$$
\begin{aligned}
N_{\mathrm{A}}^{*} & =E_{\mathrm{A}}^{*} /\left(W_{\mathrm{A}} T\right) \\
& =N_{\mathrm{A}} \alpha S_{\mathrm{A}} \beta T /\left(W_{\mathrm{A}} T\right) \propto N_{\mathrm{A}} \alpha W_{\mathrm{A}} \beta / W_{\mathrm{A}} \\
& =N_{\mathrm{A}} \alpha \beta .
\end{aligned}
$$

This now allows us to express the population growth rate for the expanding species as

$$
\begin{aligned}
R_{\text {expansion }} & =\partial \pi / \partial T=\left(\pi^{*}-\pi\right) / \pi \\
& =\left(N_{\mathrm{A}}^{*} / K\right) /\left(N_{\mathrm{A}} / K\right)-1 \\
& =N_{\mathrm{A}} \alpha \beta / N_{\mathrm{A}}-1=\alpha \beta-1 .
\end{aligned}
$$

Plugging in the expression for $\beta$ obtained earlier gives the population growth rate for the expanding species (A)

$$
R_{\text {expansion }} \propto \alpha /(\alpha \pi+1-\pi)-1 .
$$

As $\alpha$ denotes the magnitude of an evolutionary advantage of the growing species, $\alpha-1$ corresponds to the maximum population growth rate when resources are unlimited.

Similarly, the rate of decline for the declining species (B) is given by

$$
R_{\text {decline }}=\beta-1 \propto 1 /(\alpha \pi+1-\pi)-1,
$$

where $\pi$ is the relative abundance of the growing species.

From these expressions we see that the rates of expansion and decline only depend on how much trophic energy species control at the beginning and on the evolutionary advantage $\alpha$. If, as we argue, $\alpha$ does not depend on body mass, the rest of the equation does not depend on body mass. This offers a mechanistic explanation for evolution under the Red Queen, demonstrating that species' expansion (and decline) rates can be independent of body size.

Patterns of Expansion and Saturation.-Unimodal hat-like patterns of species' rise and decline have been commonly observed in the terrestrial and marine fossil record (Jernvall and Fortelius 2004; Foote et al. 2007; Liow and Stenseth 2007; Tietje and Kiessling 2013) and in living clades (Lim and Marshall 2017). These unimodal patterns are fully compatible with the Red Queen's hypothesis (Zliobaite et al. 2017), which prescribes continuous deterioration of species' effective environment due to competition.

In previous work (Zliobaite et al. 2017), we attributed unimodality to evolutionary inertia - a memory-like process that pushes species' expansion or decline through multiple time steps. Indeed, the first interpretation of the Red Queen's hypothesis suggests that once an evolutionary advantage has been acquired, the population growth will be stopped by other species acquiring counteradvantages. Yet even if no counterevolutionary advance follows from other species, over many steps the expansion resulting from an evolutionary improvement must saturate, as the new species will approach the carrying-capacity limit. This generic principle is known as logistic growth in ecology (Verhulst 1838; Pearl and Reed 1920).

Our proportional prize model for species expansion is not exactly the same as logistic growth, neither it is the same as the LotkaVolterra competitive model. Our intuition is that the fossil record will not have enough resolution to distinguish between these models in a data-driven way, and selecting the best fit is not the intention here, but rather making a point about saturation. In that sense, either of the models generate $S$-shaped curves that show saturation. The main difference is that logistic growth only considers limited carrying capacity without competition across species, while the Lotka-Volterra competitive model considers direct impact of one species on another. The proportional prize model considers no direct confrontation, but there is competition over resources. For interested readers, a more detailed comparison of the three models is presented in Appendix 3.

Figure 1 visualizes how populations would change over time following equations (1) and (2), assuming the proportional prize contest. Because no further evolution happens during the period being analyzed, all the observed growth and decline is due to inertia following 
Expanding species (A)

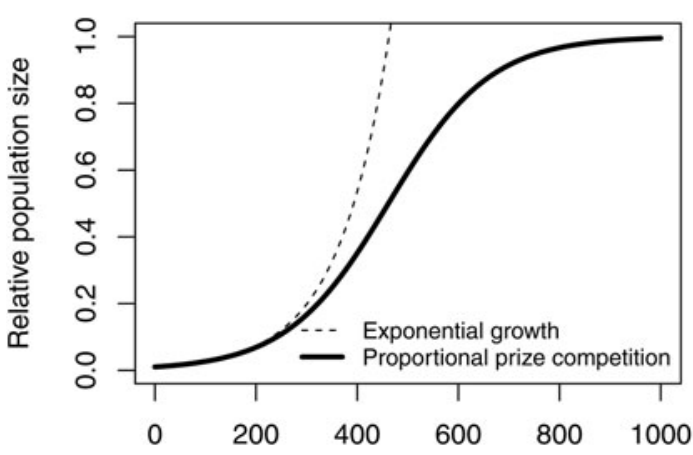

Time step

Declining species (B)

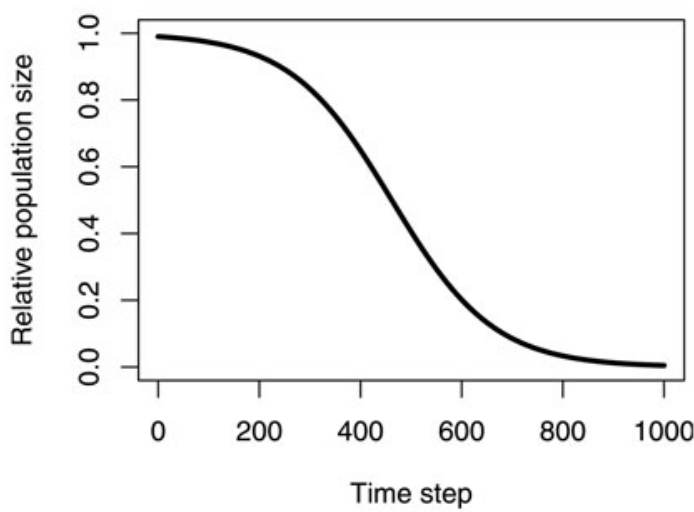

FIGURE 1. Population growth (A) and decline (B) as a proportional prize competition. Equations (1) and (2) are visualized with the evolutionary advantage parameter $\alpha=1.01$.

the initial acquisition of an evolutionary advantage. We see that the advantaged species first expands rapidly, but after a while expansion saturates, even if there is no counteraction from the competitor. Meanwhile, species B declines, following the same pattern in reverse.

In Figure 1A, population growth at the beginning looks indistinguishable from exponential growth, which would hold if unlimited resources were available. Because the population of species $A$ is very small at the beginning relative to the population of species $\mathrm{B},{ }^{*}$ there

*This assumes that A branched off from B or another parent species just recently and thus is initially small relative to the size of $B$. are plenty of opportunities for A to gain energy previously controlled by $B$, even with a small evolutionary advantage. This makes resources effectively unlimited for A. As the population of species A grows larger, competition against the other species turns into within-species competition, and the growth saturates. The shape of the expansion curve and the time of saturation here depend on the initial populations' sizes, and the magnitude of the evolutionary advantage, but not on body size.

Under this model, the initial magnitude of the evolutionary advantage determines when the growth will saturate. Sigmoid growth and saturation, however, do not explain the hat-like patterns. S-shaped growth only explains the rise of a species and its arrival at its peak population. For a decline to occur, new species need to enter the system.

Patterns of Species Rise and Decline and Their Relation to Body Size.-We demonstrate what potentially happens when new species enter the system via stochastic simulation. We build the simulation with the same proportional prize contest model, where the setting is now for multiple species over multiple time points. Mechanistic details of this simulation are as noted in the following paragraphs.

Suppose that at any point in time, individuals of existing species randomly evolve evolutionary advantages. In this simulation, evolutionary advantages are sampled from the Gaussian distribution with unit mean and variance 0.1 . This means that evolutionary advantage can occasionally be a disadvantage (when it is $<1$ ). The metabolic rate here is assumed to be $W=M^{0.75}$. To keep evolutionary advantages comparable, a fixed share of the maximal permissible population density (which is proportional to $M^{0.75}$ ) acquires an evolutionary advantage at a time to make a new species (e.g., $1 \%$ of mice acquire an advantage as well as $1 \%$ of elephants). We consider each species to belong to one of four body-mass classes, each by an order of magnitude larger than the previous one (imagine species of four types: $1 \mathrm{~kg}, 10 \mathrm{~kg}, 100 \mathrm{~kg}$, and $1000 \mathrm{~kg}$ ). The body mass for each new species is drawn uniformly at random. To effectively simulate deteriorating environment (zero-sum game) at each time step, the resource intake speeds of 
all species alive in the model are rescaled such that the average speed for the whole ecosystem is 1 . The scripts of our simulations are posted on GitHub.*

Figure 2 depicts the dynamics of population rise and decline over 1000 time steps of one simulation run. We see the patterns that are comparable across simulated body-size classes; they are mostly symmetric with respect to their peaks, although asymmetries (e.g., steeper decline than rise) are possible and occur. The patterns are unimodal, because as new species randomly enter, the environment effectively deteriorates, it never improves. Following the modeling definition of an evolutionary unit, one species evolves an advantage only once in its lifetime, so there is no way to overcome the deteriorating environment (with the next advance, a species will be a new species). In this system, species continue toward the peak from the initial inertia. This offers a simple mechanistic explanation for the future saturation (and eventual decline) that is inscribed at the time of origination of a species. It is an alternative, or perhaps a complement, to the "seed of decline" hypothesis (Fortelius et al. 2014), wherein competition from rapidly evolving (and therefore expanding) young species has been invoked to explain species decline patterns in the fossil record.

Multimodality within one species would require a second evolutionary step within the species, stochastic changes in the environment, phenotypic plasticity, or flexibility in species ecology. In the mammalian fossil record, omnivores have appeared to be the most multimodal (Jernvall and Fortelius 2004); this is attributed to their flexibility - they can change their mode of life without needing to evolve new traits.

As for body size, Figure 3 shows summary statistics of the duration of species, peak relative abundances, and energy controlled by species within each body-mass category from our simulation over 10,000 time steps. We can see from the plots that all three characteristics vary considerably, but by and large the distributions do not differ across simulated body-size classes. This is in line with the scaling arguments

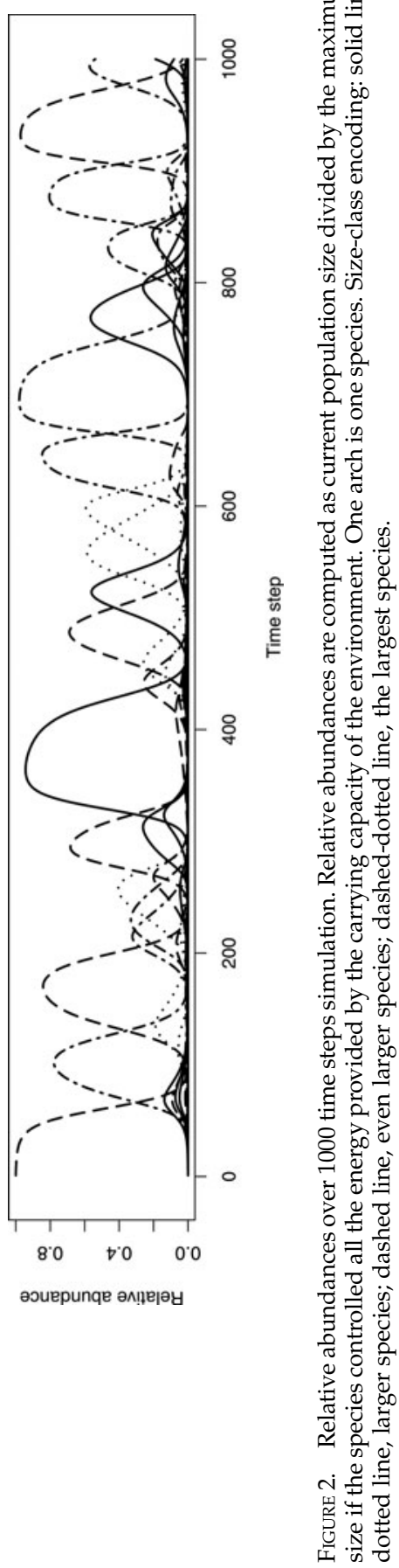

*https://github.com/zliobaite/RedQueen2. 
(A)

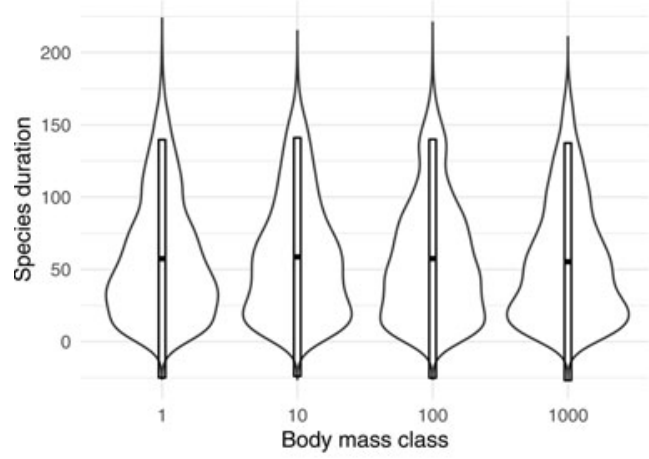

(B)

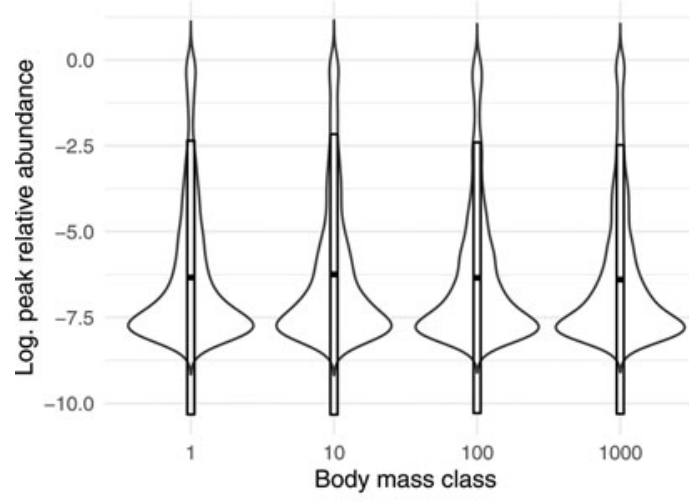

(C)

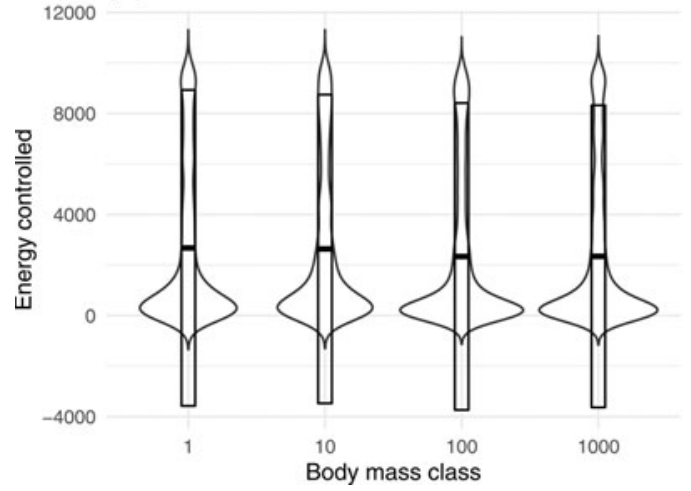

FIGURE 3. Distribution of average species' duration (A), maximum relative abundance (at the "peak"; $B$ ), and average energy controlled (as a fraction of the total carrying capacity; C) per species over 10,000 time steps.

presented earlier and suggests that in the Red Queen's domain, species of different body sizes theoretically can be equally competitive.

What If Metabolism Did Not Scale with Body Mass?-We argued that metabolic scaling allows different body sizes to be accommodated in the Red Queen's realm. Let us finish with a speculative counterfactual; What if metabolic rate was independent of body size? It is well known that animals cannot scale isometrically. If all body sizes operated at the same physiological speed, many would either overheat or starve, failing to keep up the speed of foraging with the speed of metabolism. But for the time being, let us imagine a Red Queen world in which physiological time is equal to real time, generation lengths are all equal, and energy consumption per unit of body mass is the same for all.

Such a world should, in principle, generate reverse Cope's rule patterns. At times when resources are not fully exploited, large species would grab more resources over time, because they can generate more energy storage capacity over the same time. At times when resources are fully exploited, small species would be able to enter and coexist with large species. Yet, on average, large species would reach higher relative abundances; thus energy equivalence would no longer hold. It would be a strange world, perhaps even more competitive, even more sensitive to environmental shocks, and perhaps it would accommodate less species diversity.

\section{Conclusion}

We ask under what evolutionary mechanisms large and small species can potentially be equally competitive in the Red Queen's domain. Our analysis suggests that, at least theoretically, such mechanisms exist. Individuals operate in physiological time, but need to compete in real time. The Red Queen balances between real and physiological time, such that the genetically cohesive species (or metapopulations) become the essential units of adaptive evolution. Even though small species have shorter generation lengths, they do not take over, because under zero-sum game conditions, expansive energy becomes available so slowly that generation length becomes irrelevant. Even though large species use energy more efficiently and can maintain more biomass with the same amount of energy, they cannot build more biomass with it. That is why, under tight competition, a range of body sizes remain competitive. 
This study revisits two sets of characteristic patterns commonly observed in the fossil record. We demonstrate that trends in average body mass within clades in the fossil record, known as Cope's rule, are expected to arise as a result of a gradual transition from partially exploited to fully exploited resources. Transitioning from relatively unlimited to fully exploited resources dilutes the advantage of shorter generation length and makes a wide range of body sizes competitive.

Our analysis also offers an integrative explanation for the hat-like patterns of species' rise and decline. Such patterns can emerge following a single evolutionary advantage per species. The patterns are unimodal, because as new species randomly enter, the effective environment effectively deteriorates and never improves. If species evolve an advantage only once in a lifetime, there is no way to overcome this deterioration. The initial growth saturates as across-species competition turns into within-species competition. The decline starts as more and more new species enter the scene and the environment effectively deteriorates. While the process may look more deterministic, a random component is nevertheless present, as new species are entering largely at random. This perspective on hat-like patterns offers a simpler explanation than earlier research and allows us to trace back the law of constant extinction (Van Valen 1973) and the law of constant peaking (Zliobaite et al. 2017) to an underlying principle of random origination.

Our study portrays a simplified version of evolutionary and demographic processes. The omission here of many fine ecological and evolutionary details is intentional, aiming at the simplest generic, mechanistic explanations of macroevolutionary patterns that can be derived from population processes. Needless to say, our work does not exclude alternative explanations invoking macrolevel processes but could be taken to suggest that such explanations may be unnecessary. Lack of observational data is similarly intentional. The patterns that we are analyzing are well studied and well established, while interpretations are not yet settled. We hope that our study offers a unifying perspective and a set of interpretations to consider, challenge, and refine.

\section{Acknowledgments}

We thank S. V. Haugan for an insightful discussion following our blog post that raised probing questions and provided inspiration for this analysis. We thank D. Polly, Ch. Marshall, and an anonymous reviewer for constructive criticism and helpful suggestions. Research leading to these results was partially supported by the Academy of Finland grant to I.Ž. (no. 314803).

\section{Literature Cited}

Alroy, J. 1998. Cope's rule and the dynamics of body mass evolution in North American fossil mammals. Science 280:731-734.

Ballesteros, F., V. Martinez, B. Luque, L. Lacaa, E. Valor, and A. Moya. 2018. On the thermodynamic origin of metabolic scaling. Science Reports 8:1448.

Banavar, J., M. Moses, J. Brown, J. Damuth, A. Rinaldo, R. Sibly, and A. Maritan. 2010. A general basis for quarter-power scaling in animals. Proceedings of the National Academy of Sciences USA 107:15816-15820.

Bribiesca, R., L. Herrera-Alsina, L. Sanchez-Gonzalez, and J. Schondube. 2019. Body mass as a supertrait linked to abundance and behavioral dominance in hummingbirds: a phylogenetic approach. Ecology and Evolution 9:1623-1637.

Brown, J. 1995. Macroecology. University of Chicago Press, Chicago. Brown, J., J. Gillooly, A. Allen, V. Savage, and G. West. 2004. Toward a metabolic theory of ecology. Ecology 85:1771-1789.

Brown, J. H., and B. A. Maurer. 1986. Body size, ecological dominance and Cope's rule. Nature 324:248-250.

Cason, T., W. Masters, and R. Sheremeta. 2020. Winner-take-all and proportional-prize contests: theory and experimental results. Journal of Economic Behavior and Organization 175:314-327.

Clauset, A., and D. Erwin. 2008. The evolution and distribution of species body size. Science 321:399-401.

Cunningham, A., J. Lennox, and J. Ross, eds. 2001. Biofilms: the hypertextbook. https://www.cs.montana.edu/webworks/ projects/stevesbook/index.html, accessed 20 December 2018.

Damuth, J. 1981. Population density and body size in mammals. Nature 290:699-700.

Damuth, J. 1987. Interspecific allometry of population density in mammals and other animals: the independence of body mass and population energy-use. Biological Journal of the Linnean Society 31:193-246.

Damuth, J. 2007. A macroevolutionary explanation for energy equivalence in the scaling of body size and population density. American Naturalist 169:621-631.

Damuth, J., and B. MacFadden, eds. 1990. Body size in mammalian paleobiology: estimation and biological implications. Cambridge University Press, Cambridge.

DeLong, J., J. Okie, M. Moses, R. Sibly, and J. Brown. 2010. Shifts in metabolic scaling, production, and efficiency across major evolutionary transitions of life. Proceedings of the National Academy of Sciences USA 107: 12941-12945.

de Megalhaes, J. P., J. Costa, G. M. Church. 2007. An analysis of the relationship between metabolism, developmental schedules, and longevity using phylogenetic independent contrasts. Journals of Gerontology, series A: 62:149-160.

Dobson, F., and M. Oli. 2008. The life histories of orders of mammals: fast and slow breeding. Current Science 95:862-865.

Fenchel, T. 1974. Intrinsic rate of natural increase: the relationship with body size. Oecologia 14:317-326. 
Foote, M., J. Crampton, A. Beu, B. Marshall, R. Cooper, P. Maxwell, and I. Matcham. 2007. Rise and fall of species occupancy in Cenozoic fossil mollusks. Science, 318:1131-1134.

Fortelius, M. 1985. Ungulate cheek teeth: developmental, functional and evolutionary interrelations. Acta Zoologica Fennica 180:1-76.

Fortelius, M., J. Eronen, F. Kaya, H. Tang, P. Raia, and K. Puolamäki. 2014. Evolution of Neogene mammals in Eurasia: environmental forcing and biotic interactions. Annual Reviews of Earth and Planetary Science 42:579-604.

Gillman, M. 2007. Evolutionary dynamics of vertebrate body mass range. Evolution 61:685-693.

Ginzburg, L., and M. Colyvan. 2004. Ecological orbits: how planets move and populations grow. Oxford University Press Kindle edition.

Glazier, D. 2014. Metabolic scaling in complex living systems. Systems 2:451-540.

Glazier, D. 2015. Is metabolic rate a universal "pacemaker" for biological processes? Biological Reviews 90:377-407.

Glazier, D. 2018. Rediscovering and reviving old observations and explanations of metabolic scaling in living systems. Systems 6:4.

Hallam, A. 1978. How rare is phyletic gradualism and what is its evolutionary significance? Evidence from Jurassic bivalves. Paleobiology 4:16-25.

Hamilton, M., A. Davidson, R. Sibly, and J. Brown. 2011. Universal scaling of production rates across mammalian lineages. Proceedings of the Royal Society of London B 278:560-566.

Harrison, J. F. 2017. Do performance-safety tradeoffs cause hypometric metabolic scaling in animals? Trends in Ecology and Evolution 32:653-664.

Hone, D., and M. Benton. 2005. The evolution of large size: how does Cope's rule work? Trends in Ecology and Evolution 20:4-6.

Huang, S., J. Eronen, Ch. Janis, J. Saarinen, D. Silvestro, and S. Fritz. 2017. Mammal body size evolution in North America and Europe over 20 Myr: similar trends generated by different processes. Proceedings of the Royal Society of London B 284:20162361.

Hulbert, A., R. Pamplona, R. Buffenstein, and W. Buttemer. 2007. Life and death: metabolic rate, membrane composition, and life span of animals. Physiological Reviews 87:1175-1213.

Jablonski, D. 1996. Body size and macroevolution. Pp. 256-289 in D. Jablonski, D. Erwin, and J. Lipps, eds. Evolutionary palaeobiology. University of Chicago Press, Chicago.

Jablonski, D. 1997. Body-size evolution in Cretaceous molluscs and the status of Cope's rule. Nature 385:250-252.

Jernvall, J., and M. Fortelius. 2004. Maintenance of trophic structure in fossil mammal communities: site occupancy and taxon resilience. American Naturalist 164:614- 624.

Jetz, W., Ch. Carbone, J. Fulford, and J. Brown. 2004. The scaling of animal space use. Science 306:266-268.

Keyfitz, N., and H. Caswell. 2005. Applied mathematical demography, $3^{\text {rd }}$ ed. Springer, New York.

Kirkwood, T. 1977. Evolution of ageing. Nature 270:301-304.

Kleiber, M. 1932. Body size and metabolism. Hilgardia 6:315-351.

Lim, J., and Ch. Marshall. 2017. The true tempo of evolutionary radiation and decline revealed on the Hawaiian archipelago. Nature 543:710-713.

Lindstedt, S., and W. Calder III. 1981. Body size, physiological time, and longevity of homeothermic animals. Quarterly Review of Biology 56:1-16.

Liow, L. H., and N. Chr. Stenseth. 2007. The rise and fall of species: implications for macroevolutionary and macroecological studies. Proceedings of the Royal Society of London B 274: 2745-2752.

Loewe, L., and W. G. Hill. 2010. The population genetics of mutations: good, bad and indifferent. Philosophical Transactions of the Royal Society of London B 365:1153-1167.

MacArthur, R., and E. Wilson. 1967. The theory of island biogeography. Princeton University Press, Princeton, N.J.

Marquet, P. 2002. Of predators, prey, and power laws. Science 318:1131-1134.
Marquet, P., S. Navarrete, and J. Castilla. 1995. Body size, population density, and the energetic equivalence rule. Journal of Animal Ecology 64:325-332.

Maurer, B., J. Brown, and R. Rusler. 1992. The micro and macro in body size evolution. Evolution 46:939-953.

May, R. 1988. How many species are there on Earth? Science 241:1441-1449.

McCoy, M., and J. Gillooly. 2008. Predicting natural mortality rates of plants and animals. Ecology Letters 11:710-716.

Nee, S., A. F. Read, J. J. Greenwood, and P. H. Harvey. 1991. The relationship between abundance and body size in British birds. Nature 351:312-313.

Pearl, R. 1928. The rate of living. Knopf, New York.

Pearl, R., and L. Reed. 1920. On the rate of growth of the population of the United States. Proceedings of the National Academy of Sciences USA 6:275.

Pedersen, R., S. Faurby, and J. Svenning. 2017. Shallow size-density relations within mammal clades suggest greater intra-guild ecological impact of large-bodied species. Journal of Animal Ecology 86:1205-1213.

Peters, R. 1983. Ecological implications of body size. Cambridge University Press, Cambridge.

Pianka, E. R. 1970. On r- and K-selection. American Naturalist 104:592-597.

Pineda-Munoz, S., A. Evans, and J. Alroy. 2016. The relationship between diet and body mass in terrestrial mammals. Paleobiology 42:659-669.

Polishchuk, L., and V. Tseitlin. 1999. Scaling of population density on body mass and a number- size trade-off. Oikos 99:544-556.

Polishchuk, L. V., and J. L. Blanchard. 2019. Uniting discoveries of abundance-size distributions from soils and seas. Trends in Ecology and Evolution 34:2-5.

Polly, P. D. 1998. Cope's rule. Science 282:47.

Polly, P. D., J. Fuentes-Gonzalez, A. M. Lawing, A. K. Bormet, and R. G. Dundas. 2017. Clade sorting has a greater effect than local adaptation on ecometric patterns in Carnivora. Evolutionary Ecology Research 18:61-95.

Raia, P., and M. Fortelius. 2013. Cope's law of the unspecialized, Cope's rule, and weak directionality in evolution. Evolutionary Ecology Research 15:747-756.

Raia, P., F. Carotenuto, F. Passaro, D. Fulgione, and M. Fortelius. 2012. Ecological specialization in fossil mammals explains Cope's rule. American Naturalist 179:328-337.

Rubner, M. 1908. Das Problem det Lebensdaur und seiner beziehunger zum Wachstum und Ernarnhung. Oldenberg, Munich.

Salguero-Gomez, R., O. Jones, E. Jongejans, S. Blomberg, D. Hodgson, C. Mbeau-Ache, P. Zuidema, H. de Kroon, and Y. Buckley. 2016. Fast-slow continuum and reproductive strategies structure plant life-history variation worldwide. Proceedings of the National Academy of Sciences USA 113:230-235.

Savage, V., E. Deeds, and W. Fontana. 2008. Sizing up allometric scaling theory. PLoS Computational Biology 4:e1000171.

Schmidt-Nielsen, K. 1997. Animal physiology, $5^{\text {th }}$ ed. Cambridge University Press, Cambridge.

Schmidt-Nielsen, K. 1984. Scaling: why is animal size so important? Cambridge University Press, Cambridge.

Shipley, L. A., J. E. Gross, D. E. Spalinger, N. T. Hobbs, and B. A. Wunder. 1994. The scaling of intake rate in mammalian herbivores. American Naturalist 143:1055-1082.

Sibly, R., and Brown, J. 2009. Mammal reproductive strategies driven by offspring mortality-size relationships. American Naturalist 173:E185-E199.

Simpson, G. G. 1944. Tempo and mode in evolution. Columbia University Press, New York.

Simpson, G. G. 1961. Principles of animal taxonomy. Columbia University Press, New York. 
Simpson, G. G. 1953. Major features of evolution. Columbia University Press, New York.

Smith, F., A. Boyer, J. Brown, D. Costa, T. Dayan, S. Ernest, A. R. Evans, et al.. 2010. The evolution of maximum body size of terrestrial mammals. Science 330:1216-1219.

Southwood, T., R. May, M. Hassell, and G. Conway. 1974. Ecological strategies and population parameters. American Naturalist 108:791-804.

Speakman, R. 2005. Body size, energy metabolism and lifespan. Journal of Experimental Biology 208:1717-1730.

Stanley, S. 1973. An explanation for Cope's rule. Evolution 27:1-26.

Stephens, Ph., M. Vieira, S. Willis, and Ch. Carbone. 2019. The limits to population density in birds and mammals. Ecology Letters 22:654-663.

Tidiere, M., J. M. Gaillard, V. Berger, D. Mueller, L. Lackey, O. Gimenez, M. Clauss, et al.. 2016. Comparative analyses of longevity and senescence reveal variable survival benefits of living in zoos across mammals. Science Reports 6:36361.

Tietje, M., and W. Kiessling. 2013. Predicting extinction from fossil trajectories of geographical ranges in benthic marine molluscs. Journal of Biogeography 40:790-799.

Van Valen, L. 1971. Adaptive zones and the orders of mammals. Evolution 25:420-428.

Van Valen, L. 1973. A new evolutionary law. Evolutionary Theory 1:1-30.

Van Valen, L. 1976. Energy and evolution. Evolutionary Theory 1:179-229.

Van Valen, L. 1980. Evolution as zero-sum game for energy. Evolutionary Theory 4:289-300.

Van Valkenburgh, B. 2009. Costs of carnivory: tooth fracture in Pleistocene and Recent carnivorans. Biological Journal of the Linnean Society 96:68-81.

Verhulst, P. 1838. Notice sur la loi que la population poursuit dans son accroissement. Correspondance mathématique et physique 10:113-121.

Virot, E., G. Ma, C. Clanet, and S. Jung. 2017. Physics of chewing in terrestrial mammals. Science Reports 7:43967.

Walker, T., and J. Valentine. 1984. Equilibrium models of evolutionary species diversity and the number of empty niches. American Naturalist 124:887-899.

West, G., J. Brown, and B. Enquist. 1997. A general model for the origin of allometric scaling laws in biology. Science 276:122-126.

White, C., and M. Kearney. 2014. Metabolic scaling in animals: methods, empirical results, and theoretical explanations. Comprehensive Physiology 4:231-256.

White, E. P., S. K. M. Ernest, A. J. Kerkhoff, and B. J. Enquist. 2007. Relationships between body size and abundance in ecology. Trends in Ecology and Evolution 22:323-330.

Woodward, G., B. Ebenman, M. Emmerson, J. M. Montoya, J. M. Olesen, A. Validog, and P. H. Warren. 2005. Body size in ecological networks. Trends in Ecology and Evolution 20:402-409.

Yeakel, J. D., Ch. P. Kempes, and S. Redner. 2018. Dynamics of starvation and recovery predict extinction risk and both Damuth's law and Cope's rule. Nature Communications 9:657.

Zliobaite, I., M. Fortelius, and N. Chr. Stenseth. 2017. Reconciling taxon senescence with the Red Queen's hypothesis. Nature 552:92-95.

\section{Appendix 1. The Adaptive Zone: Interpretations and Historical Remarks}

The adaptive zone is an elusive concept, and its interpretation requires considering the historical context in which it was developed. The adaptive zone was first introduced by Simpson in his classic Tempo and Mode in Evolution (1944) as a partly arbitrary concept necessary for understanding the evolution of adaptation. For him, the adaptive zone was a fundamental and pervasive feature of the living world: "Similarly, the whole animal kingdom can be viewed as occupying a complex of larger and smaller adaptive zones, each definable in joint terms of the environment and of the organisms in them. The convenience of designating these in formal taxonomic terms ('canid zone', 'felid zone' and so forth) arises from the approximation of adaptive, structural, and phylogenetic phenomena. Because of the widespread occurrence of convergence, this is only a convenience, not an equivalence" (Simpson 1944: p. 190).

In Simpson's Major Features of Evolution (1953), the concept had matured, and he added a footnote to this effect: "I now find part of my earlier discussion of this subject ... somewhat confusing in this respect" (Simpson 1953: p. 202, note 2). He downplayed his earlier, rather defensive discussion of the arbitrariness of adaptive zones and emphasized instead their dynamic and interactive nature: "It is essential to remember that what we are talking about here is not a geographic, physical, or even in the broadest sense environmental zone but an adaptive zone, representing a characteristic reaction and mutual relationship between environment and organism, a way of life and not a place where life is led" (Simpson 1953: pp. 201-202. He again emphasized that the concept was not taxonomic: "Although this adaptive relationship correlates with taxonomy as suggested, it is not confined or defined by taxonomy" (Simpson 1953: p. 200).

Van Valen (1971) clearly understood these nuances, but in order to make macroevolutionary analyses operational, he used taxonomic units as an approximation to adaptive zones. Thus, he empirically treated higher taxa as adaptive zones. The original law paper (Van Valen 1973) never explicitly discusses taxonomic versus ecologic interpretations of an adaptive zone, but from his interpretations of the law, it is clear that he means ecology: "The probability of extinction of a taxon is then effectively independent of its age. This suggests a randomly acting process. But the probability 
is strongly related to adaptive zones. This shows that a randomly acting process cannot be operating uniformly. How can it be that extinction occurs randomly with respect to age but nonrandomly with respect to ecology?" (Van Valen 1973: p. 17).

Van Valen (1971) made explicit an operational challenge that is relevant in the present context: the consideration of competitive interactions. "I therefore restrict 'adaptive zone' to its environmental meaning, i.e. some part of the resource space together with relevant predation and parasitism, and introduce as a technical term way of life, with its everyday meaning, for the interaction. 'Way of life' is also broader than 'adaptive zone' in that competitive interactions are included. We must exclude the latter from the concept of adaptive zone, or else we can't even consider the possibility of two taxa competing within the same adaptive zone" Van Valen (1971: p. 241).

Adaptive zones are an interpretive approximation of a complicated and multidimensional real-world structure comprised of fuzzy entities with porous boundaries, and implementing them in a model will necessarily have to involve arbitrary choices. At the same time, Van Valen's concept of an adaptive zone as an entity within which competition occurs is quite straightforward from a modeling perspective and we have adopted that perspective here. We understand an adaptive zone as the field in which competition occurs between species clumped into eco-evolutionary entities by their shared ways of life or resource use and model it accordingly. An adaptive zone, thus, cannot exist unoccupied.

\section{Appendix 2. Scaling Relationships with Adjustable Metabolic Rate}

Table A1 presents a summary of the scaling argument with the possibility of plugging in one's preferred metabolic scaling exponent $x$.

\section{Appendix 3. Comparing the Proportional \\ Prize Contest, Logistic Growth, and Lotka-Volterra Competitive Models}

Exponential Growth.-If resources are unlimited and there are no competitive interactions between species, maximum population growth is realized at every time step and the population growth rate is exponential, given by the following differential equation:

$$
\partial \pi / \partial T=\pi r_{\max }
$$

TABLE A1. Scaling exponents with adjustable metabolic scaling exponent.

\begin{tabular}{|c|c|c|c|}
\hline Scaling of: & Theoretical argument & $\begin{array}{l}\text { Scaling } \\
\text { relationship }\end{array}$ & $\begin{array}{l}\text { Working } \\
\text { version }\end{array}$ \\
\hline Metabolic rate per capita & Assume (Kleibner), but feel free to choose your $x$ & $M^{x}$ & $M^{0.75}$ \\
\hline Energy per species & Assume (Red Queen) & $M^{0}$ & $M^{0}$ \\
\hline Population density & $\begin{array}{l}\text { Number of individuals that can be supported with constant } \\
\text { energy and given metabolic rate, } M^{0} / M^{x}\end{array}$ & $M^{-x}$ & $M^{-0.75}$ \\
\hline $\begin{array}{l}\text { Energy consumption per } \\
\text { gram of tissue }\end{array}$ & Metabolic rate divided by body mass, $M^{x} / M^{1}$ & $M^{x-1}$ & $M^{-0.25}$ \\
\hline Durability of an organism & Assume (heartbeats, breaths, chews) & $M^{0}$ & $M^{0}$ \\
\hline Longevity of an organism & $\begin{array}{l}\text { Durability divided by the speed of energy consumption per } \\
\text { gram of tissue, } M^{0} / M^{x-1}\end{array}$ & $M^{1-x}$ & $M^{0.25}$ \\
\hline (Ecological) life expectancy & Proportional to maximum longevity & $M^{1-x}$ & $M^{0.25}$ \\
\hline Reproductive lifetime & Proportional to maximum longevity & $M^{1-x}$ & $M^{0.25}$ \\
\hline $\begin{array}{l}\text { Death rates in nongrowing } \\
\text { population }\end{array}$ & Inverse of life expectancy, $1 / M^{1-x}$ & $M^{x-1}$ & $M^{-0.25}$ \\
\hline $\begin{array}{l}\text { Birth rates in nongrowing } \\
\text { population }\end{array}$ & Equal to death rates & $M^{x-1}$ & $M^{-0.25}$ \\
\hline Biomass per species & Population density times body mass, $M^{-x} M^{1}$ & $M^{1-x}$ & $M^{0.25}$ \\
\hline $\begin{array}{l}\text { Biomass over all }{ }^{\mathrm{a}} \text { species in } \\
\text { an ecosystem }\end{array}$ & Constant with respect to body size & $M^{0}$ & $M^{0}$ \\
\hline $\begin{array}{l}\text { Number of species in an } \\
\text { ecosystem }\end{array}$ & Biomass all divided by biomass per species, $M^{0} / M^{1-x}$ & $M^{x-1}$ & $M^{-0.25}$ \\
\hline
\end{tabular}

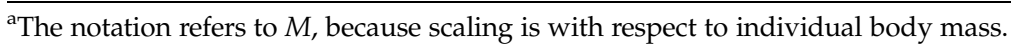


where $r_{\max }$ is the maximum population growth rate and $\pi$ is the population size at the previous time step.

Logistic Growth.-In this model resources are limited, but there are no competitive interactions across species; the inhibition to the population growth comes from within-species competition. The logistic growth is given by the following differential equation:

$$
\partial \pi / \partial T=\pi r_{\max }(1-\pi) .
$$

Proportional Prize Model.-This model considers limited carrying capacity and competition for resources, but not negative (or positive) interactions between species. If, as in our analysis, a competitive advantage is defined as $\alpha>1$, then $r_{\max }=\alpha-1$. The model is given by the following differential equation (for the expanding species):

$$
\partial \pi / \partial T=\alpha \pi /(\alpha \pi+1-\pi)-1
$$

Lotka-Volterra Competitive Model.-The twospecies model is given by the following

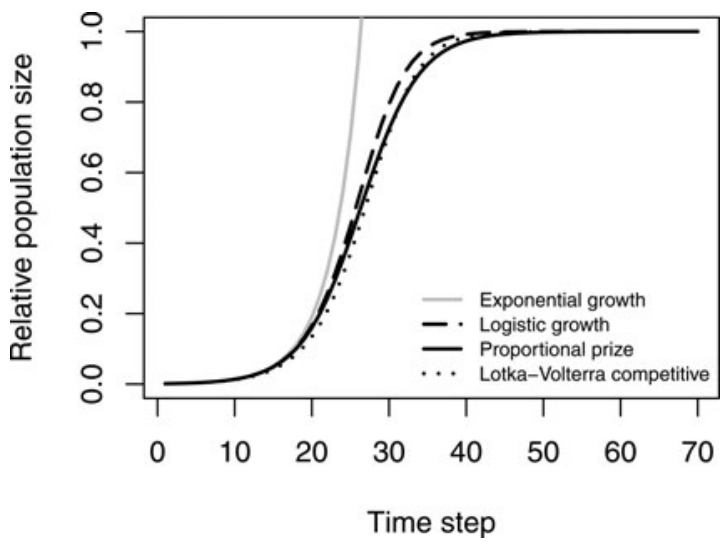

FIgURE A1. Comparison of four growth models. Parameters: for exponential growth, $r_{\max }=0.3$; for LotkaVolterra model, $\mathrm{a}=0.05$.

differential equation (for the expanding species):

$$
\partial \pi / \partial T=\pi r_{\max }(1-\pi)(1-\mathrm{a}),
$$

where $\mathrm{a}>0$ is the parameter describing the effect of species B on species A.

Figure A1 presents a visual comparison of the four models. 